\title{
Solution to the solvability problem for a class of product-type systems of difference equations
}

\section{Stevo Stević}

"Correspondence: sstevic@ptt.rs Mathematical Institute, Serbian Academy of Sciences, Knez Mihailova 36/III, Beograd, 11000, Serbia

Operator Theory and Applications Research Group, Department of Mathematics, King Abdulaziz University, P.O. Box 80203, Jeddah, 21589, Saudi Arabia

\section{Springer}

\begin{abstract}
Solution to the solvability problem for a class of product-type systems of difference equations is given by presenting explicit formulas for its solutions. In the main/most complicated cases the problem is solved by using two different methods. This is the last system, out of the three non-equivalent ones, for which an associated polynomial, which essentially determines the structure of the solutions, is of the third order, so that the paper finishes the study of this kind of two-dimensional systems.
\end{abstract}

MSC: 39A20; 39A45

Keywords: solvability problem; system of difference equations; product-type system; solutions in closed form

\section{Introduction}

Theory of difference equations is a mathematical area of great interest [1-40]. Concrete systems is one of its subareas which is of some interest nowadays. Papers [12-14] by Papaschinopoulos and Schinas are some of those which have influenced the interest. Later many others have appeared $[4,9-11,15,16,18,19,22-28,30-40]$. One of the oldest topics is finding solutions to the equations and systems $[1,5-8]$. Since the mid of 2000 s there has been increasing interest in the topic $[4,17,21-31,33-40]$. An interesting fact is that many nonlinear equations and systems are related to linear ones although they are of relatively complex forms, which usually do not suggest it on the first site. A line of the investigation related to a solvable nonlinear difference equation can be followed, for example, in $[4,17$, $21,36]$ (see also the references therein). A more complicated equation and related methods can be found in [29]. The corresponding and related systems of difference equations were studied, for example, in [23,33] and [34] (see also the references therein). Another system of interest can be found in [35]. The equations and systems in these papers are solved by employing suitable changes of variables which transform them to some linear equations and systems which are solvable.

Many equations and systems contain as special cases product-type ones [20,32]. Some methods for the study of the long-term behavior of their solutions have used directly or indirectly the equations and systems which can be solved. In [20] and [32] we studied only positive solutions to the equations and systems appearing therein, so the product-type

(c) The Author(s) 2017. This article is distributed under the terms of the Creative Commons Attribution 4.0 International License (http://creativecommons.org/licenses/by/4.0/), which permits unrestricted use, distribution, and reproduction in any medium, provided you give appropriate credit to the original author(s) and the source, provide a link to the Creative Commons license, and indicate if changes were made. 
ones, which are obtained from them by special choices of their parameters, were solvable. Applying the logarithm is a standard method for solving such equations and systems; it was, among others, also used in solving a system in [22]. In general, a more complex case is studying non-positive solutions to difference equations and systems, which is also the case for the product-type systems, since the previous method is useless because it produces multi-valued solutions. In our papers [26] and [31], we have presented a two- and a threedimensional solvable product-type system in the complex plane, respectively. Many results on the long-term behavior of solutions to the system in [31] were presented therein, while in [26] we only gave a method for solving the corresponding system. Having published these two papers has motivated us to look for other solvable product-type systems. In [37] we have managed to solve another one. The fact that the systems in [31] and [37] have been special cases of the following system

$$
z_{n}=z_{n-k_{1}}^{a} w_{n-k_{2}}^{b}, \quad w_{n}=w_{n-k_{3}}^{c} z_{n-k_{4}}^{d}, \quad n \in \mathbb{N}_{0},
$$

has motivated us to start investigating the solvability of other special cases of the system. Some equations in [29] are product-type ones, but they have additional multipliers, which suggested us to study the solvability of the generalizations of system (1) containing some multipliers. The first step in the study was made in [24]. Our further investigations suggested that closed-form formulas for solutions to product-type systems can be presented for all given values of parameters and multipliers, which for two specific systems of the form in (1) was done in [28] and [40] for the first time (in [37] and [39] general formulas were presented in the main cases, but without detailed analysis and without presenting more concrete formulas for each possible case; this type of problems does not appear for the system in [38], where its solutions are presented in all the cases). Product-type systems can be also solved by using the method which we developed in [27], but is somewhat technically complicated with respect to the one developed in [28, 37, 40]. Another class of product-type systems has been quite recently investigated in detail in [30].

This paper is devoted to the study of an extension of system (1), with the following delays $k_{1}=k_{2}=1$ and $k_{3}=k_{4}=2$, that is, of the system:

$$
z_{n}=\alpha z_{n-1}^{a} w_{n-1}^{b}, \quad w_{n}=\beta w_{n-2}^{c} z_{n-2}^{d}, \quad n \in \mathbb{N}_{0},
$$

where $a, b, c, d \in \mathbb{Z}, \alpha, \beta, z_{-2}, z_{-1}, w_{-2}, w_{-1} \in \mathbb{C}$. It is also a natural continuation of our study in $[24,27-31,37-40]$.

This is the last system, out of the three non-equivalent ones, for which an associated polynomial, which essentially determines the structure of the solutions, is of the third order, so that the paper finishes the study of this kind of systems with two dependent variables. The case when some of initial values or parameters $\alpha$ and $\beta$ are equal to zero is excluded from the consideration since such solutions are either not well defined [23] or eventually equal to zero. If a finite sum is of the form $\sum_{j=l}^{l-1} c_{j}$, for some $l \in \mathbb{Z}$, we regard that its value is zero.

\section{Auxiliary results}

The following two lemmas are useful in the investigation of product-type systems. Proofs of the first one can be found, for example, in [6] and [37]. 


\section{Lemma 1 Let}

$$
Q(t)=\sum_{j=0}^{k} c_{j} t^{j}=c_{k} \prod_{j=1}^{k}\left(t-t_{j}\right)
$$

such that $t_{i} \neq t_{j}, i \neq j$, and $c_{k} \neq 0$. Then

$$
\sum_{j=1}^{k} \frac{t_{j}^{s}}{Q^{\prime}\left(t_{j}\right)}=0
$$

for $0 \leq s \leq k-2$, and

$$
\sum_{j=1}^{k} \frac{t_{j}^{k-1}}{Q^{\prime}\left(t_{j}\right)}=\frac{1}{c_{k}} .
$$

The second lemma is well known ([6, 8], see also [28] for a more general result).

Lemma 2 Let $i \in \mathbb{N}_{0}$ and

$$
s_{n}^{(i)}(z)=1+2^{i} z+3^{i} z^{2}+\cdots+n^{i} z^{n-1}, \quad n \in \mathbb{N},
$$

where $z \in \mathbb{C}$.

Then

$$
\begin{aligned}
& s_{n}^{(0)}(z)=\frac{1-z^{n}}{1-z}, \\
& s_{n}^{(1)}(z)=\frac{1-(n+1) z^{n}+n z^{n+1}}{(1-z)^{2}}, \\
& s_{n}^{(2)}(z)=\frac{1+z-(n+1)^{2} z^{n}+\left(2 n^{2}+2 n-1\right) z^{n+1}-n^{2} z^{n+2}}{(1-z)^{3}}
\end{aligned}
$$

for every $z \in \mathbb{C} \backslash\{1\}$ and $n \in \mathbb{N}$.

\section{Main results}

Five cases will be considered separately. Before we formulate and prove our main results, note that

$$
\begin{aligned}
& z_{0}=\alpha z_{-1}^{a} w_{-1}^{b}, \quad z_{1}=\alpha^{1+a} \beta^{b} z_{-2}^{b d} z_{-1}^{a^{2}} w_{-2}^{b c} w_{-1}^{a b}, \\
& w_{0}=\beta w_{-2}^{c} z_{-2}^{d}, \quad w_{1}=\beta w_{-1}^{c} z_{-1}^{d} .
\end{aligned}
$$

Theorem 1 Assume that $a, b, d \in \mathbb{Z}, c=0, b d=0, \alpha, \beta, z_{-2}, z_{-1}, w_{-1} \in \mathbb{C} \backslash\{0\}$. Then

(a) if $a \neq 1$, the general solution to (2) is given by

$$
z_{n}=\alpha^{\frac{1-a^{n+1}}{1-a}} \beta^{\frac{1-a^{n}}{1-a}} z_{-1}^{a^{n+1}} w_{-1}^{b a^{n}}, \quad n \in \mathbb{N}
$$

and

$$
w_{n}=\beta \alpha^{d \frac{1-a^{n-1}}{1-a}} z_{-1}^{d a^{n-1}}, \quad n \geq 2 ;
$$


(b) if $a=1$, the general solution to (2) is given by

$$
z_{n}=\alpha^{n+1} \beta^{b n} z_{-1} w_{-1}^{b}, \quad n \in \mathbb{N}_{0}
$$

and

$$
w_{n}=\alpha^{d(n-1)} \beta z_{-1}^{d}, \quad n \geq 2
$$

Proof Since $c=0$, we have

$$
z_{n}=\alpha z_{n-1}^{a} w_{n-1}^{b}, \quad w_{n}=\beta z_{n-2}^{d}, \quad n \in \mathbb{N}_{0} .
$$

From the equations in (11) and the assumption $b d=0$, we get

$$
z_{n}=\alpha \beta^{b} z_{n-1}^{a}, \quad n \in \mathbb{N},
$$

and consequently

$$
z_{n}=\left(\alpha \beta^{b}\right)^{\sum_{j=0}^{n-1} a^{j}} z_{0}^{a^{n}}, \quad n \in \mathbb{N}
$$

which along with (6) yields

$$
z_{n}=\alpha^{\sum_{j=0}^{n} a^{j}} \beta^{b \sum_{j=0}^{n-1} a^{j}} z_{-1}^{a^{n+1}} w_{-1}^{b a^{n}}, \quad n \in \mathbb{N} .
$$

From (13) we easily get (7) and (9).

Using (13) in the second equality in (11), as well as the assumption $b d=0$, we obtain

$$
\begin{aligned}
w_{n} & =\alpha^{d \sum_{j=0}^{n-2} a^{j}} \beta^{1+b d \sum_{j=0}^{n-3} a^{j}} z_{-1}^{d a^{n-1}} w_{-1}^{b d a^{n-2}} \\
& =\alpha^{d \sum_{j=0}^{n-2} a^{j}} \beta z_{-1}^{d a^{n-1}}, \quad n \geq 3 .
\end{aligned}
$$

From (14) we easily get (8) and (10) for $n \geq 3$. For $n=2$, equalities (8) and (10) are directly verified.

Theorem 2 Assume that $a, c, d \in \mathbb{Z}, c \neq 0, b=0, \alpha, \beta, z_{-2}, z_{-1}, w_{-2}, w_{-1} \in \mathbb{C} \backslash\{0\}$. Then system (2) is solvable in closed form.

This theorem was essentially proved in [39], Theorem 2, (see also [39], Corollary 2, for the closed form formulas for the solutions in all the cases), since when $b=0$ system (2) is

$$
z_{n}=\alpha z_{n-1}^{a}, \quad w_{n}=\beta w_{n-2}^{c} z_{n-2}^{d}, \quad n \in \mathbb{N}_{0},
$$

which is nothing but the corresponding system in Theorem 2 in [39] with indices shifted backward for one. Hence, we omit the proof of the theorem.

Theorem 3 Assume that $a, b, c \in \mathbb{Z}, c \neq 0, d=0, \alpha, \beta, z_{-1}, w_{-2}, w_{-1} \in \mathbb{C} \backslash\{0\}$. Then system (2) is solvable in closed form. 
This theorem was essentially proved in [28], since when $d=0$ system (2) is

$$
z_{n}=\alpha z_{n-1}^{a} w_{n-1}^{b}, \quad w_{n}=\beta w_{n-2}^{c}, \quad n \in \mathbb{N}_{0},
$$

which is nothing but the corresponding system in Theorem 3.7 in [28] whose indices are shifted backward for one (see also [28], Corollary 3.8, for the closed form formulas for the solutions in all the cases). Hence, the proof of the theorem will be also omitted.

Theorem 4 Assume that $a, b, c, d \in \mathbb{Z} \backslash\{0\}, a c \neq b d, \alpha, \beta, z_{-2}, z_{-1}, w_{-2}, w_{-1} \in \mathbb{C} \backslash\{0\}$. Then system (2) is solvable in closed form.

Proof Since $\alpha, \beta, z_{-2}, z_{-1}, w_{-2}, w_{-1} \in \mathbb{C} \backslash\{0\}$, from (2) we get $z_{n} \neq 0 \neq w_{n}$ for $n \geq-2$. Hence

$$
w_{n-1}^{b}=\frac{z_{n}}{\alpha z_{n-1}^{a}}, \quad n \in \mathbb{N}_{0}
$$

and

$$
w_{n}^{b}=\beta^{b} w_{n-2}^{b c} z_{n-2}^{b d}, \quad n \in \mathbb{N}_{0},
$$

from which it follows that

$$
z_{n+1}=\alpha^{1-c} \beta^{b} z_{n}^{a} z_{n-1}^{c} z_{n-2}^{b d-a c}, \quad n \in \mathbb{N} .
$$

Let $\mu=\alpha^{1-c} \beta^{b}$,

$$
a_{1}=a, \quad b_{1}=c, \quad c_{1}=b d-a c, \quad y_{1}=1 .
$$

Then

$$
z_{n+1}=\mu^{y_{1}} z_{n}^{a_{1}} z_{n-1}^{b_{1}} z_{n-2}^{c_{1}}, \quad n \in \mathbb{N} .
$$

Equality (17) implies

$$
\begin{aligned}
z_{n+1} & =\mu^{y_{1}}\left(\mu z_{n-1}^{a_{1}} z_{n-2}^{b_{1}} z_{n-3}^{c_{1}}\right)^{a_{1}} z_{n-1}^{b_{1}} z_{n-2}^{c_{1}} \\
& =\mu^{y_{1}+a_{1}} z_{n-1}^{a_{1} a_{1}+b_{1}} z_{n-2}^{b_{1} a_{1}+c_{1}} z_{n-3}^{c_{1} a_{1}} \\
& =\mu^{y_{2}} z_{n-1}^{a_{2}} z_{n-2}^{b_{2}} z_{n-3}^{c_{2}}
\end{aligned}
$$

for $n \geq 2$, where

$$
a_{2}:=a_{1} a_{1}+b_{1}, \quad b_{2}:=b_{1} a_{1}+c_{1}, \quad c_{2}:=c_{1} a_{1}, \quad y_{2}=y_{1}+a_{1} .
$$

Assume

$$
z_{n+1}=\mu^{y_{k}} z_{n+1-k}^{a_{k}} z_{n-k}^{b_{k}} z_{n-k-1}^{c_{k}}
$$


for a $k \geq 2$ and all $n \geq k$, and

$$
\begin{aligned}
& a_{k}=a_{1} a_{k-1}+b_{k-1}, \quad b_{k}=b_{1} a_{k-1}+c_{k-1}, \quad c_{k}=c_{1} a_{k-1}, \\
& y_{k}=y_{k-1}+a_{k-1} .
\end{aligned}
$$

If we replace $n$ by $n-k$ in (17) and employ it in (20), we get

$$
\begin{aligned}
z_{n+1} & =\mu^{y_{k}}\left(\mu z_{n-k}^{a_{1}} z_{n-k-1}^{b_{1}} z_{n-k-2}^{c_{1}}\right)^{a_{k}} z_{n-k}^{b_{k}} z_{n-k-1}^{c_{k}} \\
& =\mu^{y_{k}+a_{k}} z_{n-k}^{a_{1} a_{k}+b_{k}} z_{n-k-1}^{b_{1} a_{k}+c_{k}} z_{n-k-2}^{c_{1} a_{k}} \\
& =\mu^{y_{k+1}} z_{n-k}^{a_{k+1}} z_{n-k-1}^{b_{k+1}} z_{n-k-2}^{c_{k+1}}
\end{aligned}
$$

for $n \geq k+1$, where

$$
a_{k+1}:=a_{1} a_{k}+b_{k}, \quad b_{k+1}:=b_{1} a_{k}+c_{k}, \quad c_{k+1}:=c_{1} a_{k}, \quad y_{k+1}:=y_{k}+a_{k} \text {. }
$$

Relations (18), (19), (23), (24) along with the inductive argument confirm the conjectures in (20)-(22).

Setting $k=n$ in (20) and using (6), (21) and (22), we have

$$
\begin{aligned}
z_{n+1} & =\mu^{y_{n}} z_{1}^{a_{n}} z_{0}^{b_{n}} z_{-1}^{c_{n}} \\
& =\left(\alpha^{1-c} \beta^{b}\right)^{y_{n}}\left(\alpha^{1+a} \beta^{b} z_{-2}^{b d} z_{-1}^{a^{2}} w_{-2}^{b c} w_{-1}^{a b}\right)^{a_{n}}\left(\alpha z_{-1}^{a} w_{-1}^{b}\right)^{b_{n}} z_{-1}^{c_{n}} \\
& =\alpha^{(1-c) y_{n}+(1+a) a_{n}+b_{n}} \beta^{b\left(y_{n}+a_{n}\right)} z_{-2}^{b d a_{n}} z_{-1}^{a^{2} a_{n}+a b_{n}+c_{n}} w_{-2}^{b c a_{n}} w_{-1}^{a b a_{n}+b b_{n}} \\
& =\alpha^{y_{n+2}-c y_{n}} \beta^{b y_{n+1}} z_{-2}^{b d a_{n}} z_{-1}^{a_{n+2}-c a_{n}} w_{-2}^{b c a_{n}} w_{-1}^{b a_{n+1}}
\end{aligned}
$$

for $n \in \mathbb{N}$.

The relations in (21) show that

$$
a_{k}=a_{1} a_{k-1}+b_{1} a_{k-2}+c_{1} a_{k-3}, \quad \text { for } k \geq 4 \text {. }
$$

Since $c_{1}=b d-a c \neq 0$, (26) yields

$$
a_{k-3}=\frac{a_{k}-a_{1} a_{k-1}-b_{1} a_{k-2}}{c_{1}} .
$$

This equality enables us to calculate $a_{j}$ for $j \leq 0$. It is obtained that

$$
a_{-1}=a_{-2}=0, \quad a_{0}=1
$$

(see detailed corresponding calculations in [28] and [40]) from which along with (22), it is obtained

$$
y_{-2}=y_{-1}=y_{0}=0, \quad y_{1}=1
$$


and

$$
y_{k}=\sum_{j=0}^{k-1} a_{j}, \quad k \in \mathbb{N} .
$$

Linear equation (26) can be solved, from which along with (28) a closed form formula for $a_{k}$ is obtained. Using (30) and Lemma 2, a closed form formula for $y_{k}$ is obtained. Employing such obtained formulas for $a_{k}$ and $y_{k}$ in (25), we obtain a closed form formula for the solution to (15).

From (2), we also have

$$
\begin{aligned}
& z_{n-2}^{d}=\frac{w_{n}}{\beta w_{n-2}^{c}}, \quad n \in \mathbb{N}_{0}, \\
& z_{n}^{d}=\alpha^{d} z_{n-1}^{a d} w_{n-1}^{b d}, \quad n \in \mathbb{N}_{0},
\end{aligned}
$$

and consequently

$$
w_{n+2}=\alpha^{d} \beta^{1-a} w_{n+1}^{a} w_{n}^{c} w_{n-1}^{b d-a c}, \quad n \in \mathbb{N}_{0} .
$$

As above, we get

$$
w_{n+2}=\eta^{y_{k}} w_{n+2-k}^{a_{k}} w_{n+1-k}^{b_{k}} w_{n-k}^{c_{k}}, \quad n \geq k-1,
$$

where $\eta=\alpha^{d} \beta^{1-a}, a_{k}, b_{k}$ and $c_{k}$ are defined by (16) and (21), and $y_{k}$ is defined by (22) and (29).

Setting $k=n+1$ in (34) and using (6), we get

$$
\begin{aligned}
w_{n+2} & =\eta^{y_{n+1}} w_{1}^{a_{n+1}} w_{0}^{b_{n+1}} w_{-1}^{c_{n+1}} \\
& =\left(\alpha^{d} \beta^{1-a}\right)^{y_{n+1}}\left(\beta w_{-1}^{c} z_{-1}^{d}\right)^{a_{n+1}}\left(\beta w_{-2}^{c} z_{-2}^{d}\right)^{b_{n+1}} w_{-1}^{c_{n+1}} \\
& =\alpha^{d y_{n+1}} \beta^{(1-a) y_{n+1}+a_{n+1}+b_{n+1}} z_{-2}^{d b_{n+1}} z_{-1}^{d a_{n+1}} w_{-2}^{c b_{n+1}} w_{-1}^{c a_{n+1}+c_{n+1}} \\
& =\alpha^{d y_{n+1}} \beta^{y_{n+3}-a y_{n+2}} z_{-2}^{d\left(a_{n+2}-a a_{n+1}\right)} z_{-1}^{d a_{n+1}} w_{-2}^{c\left(a_{n+2}-a a_{n+1}\right)} w_{-1}^{a_{n+3}-a a_{n+2}}
\end{aligned}
$$

for $n \in \mathbb{N}_{0}$.

As we have already mentioned, closed form formulas for $a_{k}$ and $y_{k}$ can be found, from which along with (35) it follows that (33) is solvable. It is not difficult to see that formulas (25) and (35) present a solution to system (2), from which the theorem follows.

Remark 1 Theorem 4 can be also proved by using the method in [27]. We include the proof also for the benefit of the reader and since in the theorem that follows we follow the lines of the method.

First note that

$$
\begin{aligned}
& w_{2}=\beta w_{0}^{c} z_{0}^{d}=\beta\left(\beta w_{-2}^{c} z_{-2}^{d}\right)^{c}\left(\alpha z_{-1}^{a} w_{-1}^{b}\right)^{d}=\alpha^{d} \beta^{1+c} z_{-2}^{c d} z_{-1}^{a d} w_{-2}^{c^{2}} w_{-1}^{b d} \\
& w_{3}=\beta w_{1}^{c} z_{1}^{d}=\alpha^{d(1+a)} \beta^{1+c+b d} z_{-2}^{b d^{2}} z_{-1}^{\left(a^{2}+c\right) d} w_{-2}^{b c d} w_{-1}^{a b d+c^{2}}
\end{aligned}
$$


The equalities in (36) show that $w_{2}$ and $w_{3}$ depend on all the parameters and initial values (we neglect the cases when some of the powers are zero). This fact and (2) show that the same holds for every $w_{n}, n \geq 2$, from which along with the first equation in (2) it follows that the same holds for every $z_{n}, n \geq 3$. Hence

$$
\begin{aligned}
& z_{n}=\alpha^{x_{n}} \beta^{y_{n}} z_{-2}^{a_{n}} z_{-1}^{b_{n}} w_{-2}^{c_{n}} w_{-1}^{d_{n}}, \\
& w_{n}=\alpha^{u_{n}} \beta^{v_{n}} z_{-2}^{\alpha_{n}} z_{-1}^{\beta_{n}} w_{-2}^{\gamma_{n}} w_{-1}^{\delta_{n}},
\end{aligned}
$$

for $n \geq-2$, for some sequences $x_{n}, y_{n}, a_{n}, b_{n}, c_{n}, d_{n}, u_{n}, v_{n}, \alpha_{n}, \beta_{n}, \gamma_{n}, \delta_{n}$.

Clearly, it must be

$$
\begin{aligned}
& x_{-2}=0, \quad y_{-2}=0, \quad a_{-2}=1, \quad b_{-2}=0, \quad c_{-2}=0, \quad d_{-2}=0 \text {, } \\
& u_{-2}=0, \quad v_{-2}=0, \quad \alpha_{-2}=0, \quad \beta_{-2}=0, \quad \gamma_{-2}=1, \quad \delta_{-2}=0 \text {, } \\
& x_{-1}=0, \quad y_{-1}=0, \quad a_{-1}=0, \quad b_{-1}=1, \quad c_{-1}=0, \quad d_{-1}=0, \\
& u_{-1}=0, \quad v_{-1}=0, \quad \alpha_{-1}=0, \quad \beta_{-1}=0, \quad \gamma_{-1}=0, \quad \delta_{-1}=1, \\
& x_{0}=1, \quad y_{0}=0, \quad a_{0}=0, \quad b_{0}=a, \quad c_{0}=0, \quad d_{0}=b, \\
& u_{0}=0, \quad v_{0}=1, \quad \alpha_{0}=d, \quad \beta_{0}=0, \quad \gamma_{0}=c, \quad \delta_{0}=0, \\
& x_{1}=1+a, \quad y_{1}=b, \quad a_{1}=b d, \quad b_{1}=a^{2}, \quad c_{1}=b c, \quad d_{1}=a b, \\
& u_{1}=0, \quad v_{1}=1, \quad \alpha_{1}=0, \quad \beta_{1}=d, \quad \gamma_{1}=0, \quad \delta_{1}=c .
\end{aligned}
$$

Applying (37) and (38) in (2), we get

$$
\begin{aligned}
z_{n}= & \alpha\left(\alpha^{x_{n-1}} \beta^{y_{n-1}} z_{-2}^{a_{n-1}} z_{-1}^{b_{n-1}} w_{-2}^{c_{n-1}} w_{-1}^{d_{n-1}}\right)^{a}\left(\alpha^{u_{n-1}} \beta^{v_{n-1}} z_{-2}^{\alpha_{n-1}} z_{-1}^{\beta_{n-1}} w_{-2}^{\gamma_{n-1}} w_{-1}^{\delta_{n-1}}\right)^{b} \\
= & \alpha^{a x_{n-1}+b u_{n-1}+1} \beta^{a y_{n-1}+b v_{n-1}} z_{-2}^{a a_{n-1}+b \alpha_{n-1}} z_{-1}^{a b_{n-1}+b \beta_{n-1}} \\
& \times w_{-2}^{a c_{n-1}+b \gamma_{n-1}} w_{-1}^{a d_{n-1}+b \delta_{n-1}}
\end{aligned}
$$

for $n \in \mathbb{N}_{0}$, and

$$
\begin{aligned}
w_{n}= & \beta\left(\alpha^{u_{n-2}} \beta^{v_{n-2}} z_{-2}^{\alpha_{n-2}} z_{-1}^{\beta_{n-2}} w_{-2}^{\gamma_{n-2}} w_{-1}^{\delta_{n-2}}\right)^{c}\left(\alpha^{x_{n-2}} \beta^{y_{n-2}} z_{-2}^{a_{n-2}} z_{-1}^{b_{n-2}} w_{-2}^{c_{n-2}} w_{-1}^{d_{n-2}}\right)^{d} \\
= & \alpha^{d x_{n-2}+c u_{n-2}} \beta^{d y_{n-2}+c v_{n-2}+1} z_{-2}^{d a_{n-2}+c \alpha_{n-2}} z_{-1}^{d b_{n-2}+c \beta_{n-2}} \\
& \times w_{-2}^{d c_{n-2}+c \gamma_{n-2}} w_{-1}^{d d_{n-2}+c \delta_{n-2}}
\end{aligned}
$$

for $n \in \mathbb{N}_{0}$.

Motivated by (37)-(48), we define sequences $x_{n}, y_{n}, a_{n}, b_{n}, c_{n}, d_{n}, u_{n}, v_{n}, \alpha_{n}, \beta_{n}, \gamma_{n}, \delta_{n}$ as follows:

$$
\begin{array}{ll}
x_{n}:=a x_{n-1}+b u_{n-1}+1, & u_{n}:=d x_{n-2}+c u_{n-2}, \\
y_{n}:=a y_{n-1}+b v_{n-1}, & v_{n}:=d y_{n-2}+c v_{n-2}+1, \\
a_{n}:=a a_{n-1}+b \alpha_{n-1}, & \alpha_{n}:=d a_{n-2}+c \alpha_{n-2}, \\
b_{n}:=a b_{n-1}+b \beta_{n-1}, & \beta_{n}:=d b_{n-2}+c \beta_{n-2},
\end{array}
$$




$$
\begin{array}{ll}
c_{n}:=a c_{n-1}+b \gamma_{n-1}, & \gamma_{n}:=d c_{n-2}+c \gamma_{n-2}, \\
d_{n}:=a d_{n-1}+b \delta_{n-1}, & \delta_{n}:=d d_{n-2}+c \delta_{n-2},
\end{array}
$$

for $n \in \mathbb{N}_{0}$.

Relations (39)-(44), (49)-(54) define $x_{n}, y_{n}, a_{n}, b_{n}, c_{n}, d_{n}, u_{n}, v_{n}, \alpha_{n}, \beta_{n}, \gamma_{n}, \delta_{n}$ uniquely, while $z_{n}$ and $w_{n}$ in (2) are defined uniquely by initial values $z_{-2}, z_{-1}, w_{-2}, w_{-1}$. Since $z_{n}$ and $w_{n}$ defined by (47) and (48) are solutions to (2) such that their values for $n=0,1$ are the same, it follows that they are closed form formulas for solutions to system (2). Hence, we need only to prove that systems (49)-(54) are solvable.

The condition $d \neq 0$ implies

$$
x_{n-2}=\frac{u_{n}-c u_{n-2}}{d}, \quad n \in \mathbb{N}_{0} \text {, }
$$

which along with the first equality in (49) yields

$$
u_{n+2}=a u_{n+1}+c u_{n}+(b d-a c) u_{n-1}+d, \quad n \in \mathbb{N}_{0},
$$

and also

$$
y_{n-2}=\frac{v_{n}-c v_{n-2}-1}{d}, \quad n \in \mathbb{N}_{0}
$$

which along with the first equality in (50) yields

$$
v_{n+2}=a v_{n+1}+c v_{n}+(b d-a c) v_{n-1}+1-a, \quad n \in \mathbb{N}_{0} .
$$

We also have

$$
\begin{aligned}
& a_{n-2}=\frac{\alpha_{n}-c \alpha_{n-2}}{d}, \\
& b_{n-2}=\frac{\beta_{n}-c \beta_{n-2}}{d}, \\
& c_{n-2}=\frac{\gamma_{n}-c \gamma_{n-2}}{d}, \\
& d_{n-2}=\frac{\delta_{n}-c \delta_{n-2}}{d},
\end{aligned}
$$

for $n \in \mathbb{N}_{0}$, and consequently

$$
\begin{aligned}
& \alpha_{n+2}=a \alpha_{n+1}+c \alpha_{n}+(b d-a c) \alpha_{n-1}, \\
& \beta_{n+2}=a \beta_{n+1}+c \beta_{n}+(b d-a c) \beta_{n-1}, \\
& \gamma_{n+2}=a \gamma_{n+1}+c \gamma_{n}+(b d-a c) \gamma_{n-1}, \\
& \delta_{n+2}=a \delta_{n+1}+c \delta_{n}+(b d-a c) \delta_{n-1},
\end{aligned}
$$

for $n \in \mathbb{N}_{0}$.

It is important to point out that all the transformations that we have employed transform systems (49)-(54) into equivalent ones. Therefore, there is a bijection between the sets of solutions to the original and transformed systems. 
Let

$$
A\left(t_{n}\right)=t_{n+2}-a t_{n+1}-c t_{n}+(a c-b d) t_{n-1}, \quad n \geq-1,
$$

where $\left(t_{n}\right)_{n \geq-2}$ is a sequence. Then $A$ is a linear operator, (56) can be written as $A\left(u_{n}\right)=d$, $n \in \mathbb{N}_{0}$, (58) as $A\left(v_{n}\right)=1-a, n \in \mathbb{N}_{0}$, and (63)-(66) as

$$
A\left(\alpha_{n}\right)=A\left(\beta_{n}\right)=A\left(\gamma_{n}\right)=A\left(\delta_{n}\right)=0, \quad n \in \mathbb{N}_{0} .
$$

Since the equation

$$
A\left(t_{n}\right)=0
$$

is linear of third order, it can be solved, and specially closed form formulas for $\alpha_{n}, \beta_{n}$, $\gamma_{n}, \delta_{n}$ can be obtained for the corresponding initial conditions (39)-(44). Employing the formulas for $\alpha_{n}, \beta_{n}, \gamma_{n}, \delta_{n}$ in (59)-(62), formulas for $a_{n}, b_{n}, c_{n}, d_{n}$ are obtained.

When $h \in \mathbb{R}$, the equation

$$
A\left(t_{n}\right)=h
$$

can be also solved (especially for $h=d$ and $h=1-a$ ), since a particular solution to the equation can be found in the following form:

$$
t_{n}=g_{0}+g_{1} n+g_{2} n^{2}+g_{3} n^{3}
$$

for some constants $g_{j}, j=\overline{0,3}$ (see, e.g., [1, 8]). From this and using (39)-(44), formulas for $u_{n}$ and $v_{n}$ can be found, from which along with (55) and (57) formulas for $x_{n}$ and $y_{n}$ are obtained. Using the obtained formulas for these twelve sequences in (47) and (48), we obtain closed form formulas for solutions to system (2).

\subsection{Forms of solutions to (2) for the case $a c \neq b d$}

The characteristic polynomial associated to (26) is

$$
P_{3}(\lambda)=\lambda^{3}-a \lambda^{2}-c \lambda+a c-b d,
$$

which is of the third order due to the assumption $a c \neq b d$. By using the change of variables $\lambda=t+\frac{a}{3}$, the equation $P_{3}(\lambda)=0$ becomes

$$
t^{3}+p t+q=0
$$

where $p=-\left(a^{2}+3 c\right) / 3$ and $q=\left(18 a c-27 b d-2 a^{3}\right) / 27$, which is solved by looking for solutions in the form $t=u+v$ and posing the condition $3 u v=-p$. Then $u^{3}$ and $v^{3}$ are solutions of the equation $z^{2}+q z-\frac{p^{3}}{27}=0$, from which it follows that

$$
\lambda_{j}=\frac{a}{3}+\varepsilon^{j-1} \sqrt[3]{-\frac{q}{2}-\sqrt{\frac{q^{2}}{4}+\frac{p^{3}}{27}}}+\bar{\varepsilon}^{j-1} \sqrt[3]{-\frac{q}{2}+\sqrt{\frac{q^{2}}{4}+\frac{p^{3}}{27}}}, \quad j=\overline{1,3},
$$

where $\varepsilon^{3}=1, \varepsilon \neq 1$. 
If the discriminant

$$
\Delta:=4 p^{3}+27 q^{2}
$$

is such that $\Delta \neq 0$, then all three zeros are different (if $\Delta>0$ two zeros are complex conjugate, if $\Delta<0$ all three zeros are real), whereas if $\Delta=0$, at least two zeros are equal [41].

Case $\Delta \neq 0$. Since in this case the zeros of $P_{3}$ are different, the general solution to (26) is

$$
u_{n}=\alpha_{1} \lambda_{1}^{n}+\alpha_{2} \lambda_{2}^{n}+\alpha_{3} \lambda_{3}^{n}, \quad n \in \mathbb{N}
$$

where $\alpha_{i}, i=\overline{1,3}$, are constants (in fact, (73) holds for every $n \in \mathbb{Z}$ due to the assumption $a c \neq b d$; see the relation in (27)).

Lemma 1 employed to $P_{3}$ shows that

$$
\sum_{j=1}^{3} \frac{\lambda_{j}^{l}}{P_{3}^{\prime}\left(\lambda_{j}\right)}=0, \quad \text { for } l=0,1, \quad \text { and } \quad \sum_{j=1}^{3} \frac{\lambda_{j}^{2}}{P_{3}^{\prime}\left(\lambda_{j}\right)}=1
$$

From (28), (73) and (74) it follows that

$$
\begin{aligned}
a_{n}= & \sum_{j=1}^{3} \frac{\lambda_{j}^{n+2}}{P_{3}^{\prime}\left(\lambda_{j}\right)}=\frac{\lambda_{1}^{n+2}}{\left(\lambda_{1}-\lambda_{2}\right)\left(\lambda_{1}-\lambda_{3}\right)}+\frac{\lambda_{2}^{n+2}}{\left(\lambda_{2}-\lambda_{1}\right)\left(\lambda_{2}-\lambda_{3}\right)} \\
& +\frac{\lambda_{3}^{n+2}}{\left(\lambda_{3}-\lambda_{1}\right)\left(\lambda_{3}-\lambda_{2}\right)}
\end{aligned}
$$

for $n \geq-2$.

Equalities (30) and (75) yield

$$
y_{n}=\sum_{i=0}^{n-1} \sum_{j=1}^{3} \frac{\lambda_{j}^{i+2}}{P_{3}^{\prime}\left(\lambda_{j}\right)}, \quad n \in \mathbb{N} .
$$

If $P_{3}(1) \neq 0$, that is, $(a-1)(c-1) \neq b d$, then (76) yields

$$
\begin{aligned}
y_{n}= & \frac{\lambda_{1}^{2}\left(\lambda_{1}^{n}-1\right)}{\left(\lambda_{1}-\lambda_{2}\right)\left(\lambda_{1}-\lambda_{3}\right)\left(\lambda_{1}-1\right)}+\frac{\lambda_{2}^{2}\left(\lambda_{2}^{n}-1\right)}{\left(\lambda_{2}-\lambda_{1}\right)\left(\lambda_{2}-\lambda_{3}\right)\left(\lambda_{2}-1\right)} \\
& +\frac{\lambda_{3}^{2}\left(\lambda_{3}^{n}-1\right)}{\left(\lambda_{3}-\lambda_{1}\right)\left(\lambda_{3}-\lambda_{2}\right)\left(\lambda_{3}-1\right)} .
\end{aligned}
$$

Using (74), it is easily seen that (77) holds for $n \geq-2$.

If $P_{3}(1)=0$, that is, $(a-1)(c-1)=b d$, and $P^{\prime}(1)=3-2 a-c \neq 0$, then

$$
P_{3}(\lambda)=\lambda^{3}-a \lambda^{2}-c \lambda+a+c-1=(\lambda-1)\left(\lambda^{2}+(1-a) \lambda+1-a-c\right),
$$

so that $\lambda_{1}=1$,

$$
\lambda_{2,3}=\frac{a-1 \pm \sqrt{a^{2}+2 a+4 c-3}}{2} \neq 1
$$


and

$$
y_{n}=\frac{\lambda_{2}^{2}\left(\lambda_{2}^{n}-1\right)}{\left(\lambda_{2}-\lambda_{3}\right)\left(\lambda_{2}-1\right)^{2}}+\frac{\lambda_{3}^{2}\left(\lambda_{3}^{n}-1\right)}{\left(\lambda_{3}-\lambda_{2}\right)\left(\lambda_{3}-1\right)^{2}}+\frac{n}{\left(\lambda_{2}-1\right)\left(\lambda_{3}-1\right)} .
$$

Using (74) it is easily checked that (80) holds for $n \geq-2$ (for a detailed explanation, see [40]).

Corollary 1 Assume that $a, b, c, d \in \mathbb{Z} \backslash\{0\}, \alpha, \beta, z_{-2}, z_{-1}, w_{-2}, w_{-1} \in \mathbb{C} \backslash\{0\}, a c \neq b d$ and $\Delta \neq 0$. Then the following statements hold.

(a) If $(a-1)(c-1) \neq b d$, then the general solution to system (2) is given by (25) and (35), where $\left(a_{n}\right)_{n \geq-2}$ is given by (75), $\left(y_{n}\right)_{n \geq-2}$ is given by (77), and $\lambda_{j}, j=\overline{1,3}$, are given by (71).

(b) If $(a-1)(c-1)=b d$ and $2 a+c \neq 3$, then the general solution to system (2) is given by (25) and (35), where $\left(a_{n}\right)_{n \geq-2}$ is given by (75) with $\lambda_{1}=1,\left(y_{n}\right)_{n \geq-2}$ is given by (80), $\lambda_{1}=1$, while $\lambda_{2,3}$ are given by (79).

Remark 2 As we have already mentioned, $P_{3}$ has a zero equal to 1 if and only if $(a-1)(c-$ $1)=b d$, so that (78) holds. The condition $\Delta \neq 0$ will be satisfied if and only if

$$
\left(18 a c-27(a-1)(c-1)-2 a^{3}\right)^{2} \neq 4\left(a^{2}+3 c\right)^{3} .
$$

For example, if $a=c \in \mathbb{Z} \backslash\{0,1\}$ and $b d=(a-1)^{2} \neq 0$, then $\Delta \neq 0$,

$$
P_{3}(\lambda)=\lambda^{3}-a \lambda^{2}-a \lambda+2 a-1=(\lambda-1)\left(\lambda^{2}-(a-1) \lambda+1-2 a\right),
$$

and conditions of Corollary $1(\mathrm{~b})$ are satisfied. Note that $\lambda_{1}=1$ and

$$
\lambda_{2,3}=\frac{a-1 \pm \sqrt{a^{2}+6 a-3}}{2} .
$$

Thus $P_{3}$ can have three different zeros, one of which is equal to 1 .

Case $\Delta=0$. In this case, $P_{3}$ has at least two equal zeros. We may assume that $\lambda_{2}=\lambda_{3}$. If $p \neq 0$, then $\lambda_{2}$ is not a triple zero of $P_{3}$, so the general solution to (26) is

$$
a_{n}=\hat{g}_{1} \lambda_{1}^{n}+\left(\hat{g}_{2}+\hat{g}_{3} n\right) \lambda_{2}^{n}, \quad n \in \mathbb{N},
$$

where $\hat{g}_{j}, j=\overline{1,3}$, are constants. Since the solution satisfies (28), we can find it by letting $\lambda_{3} \rightarrow \lambda_{2}$ in (75), and obtain

$$
a_{n}=\frac{\lambda_{1}^{n+2}-(n+2) \lambda_{1} \lambda_{2}^{n+1}+(n+1) \lambda_{2}^{n+2}}{\left(\lambda_{2}-\lambda_{1}\right)^{2}}, \quad n \geq-2
$$

from which along with (30), we get

$$
y_{n}=\sum_{j=0}^{n-1} \frac{\lambda_{1}^{j+2}+\left(\lambda_{2}-2 \lambda_{1}+j\left(\lambda_{2}-\lambda_{1}\right)\right) \lambda_{2}^{j+1}}{\left(\lambda_{2}-\lambda_{1}\right)^{2}}, \quad n \in \mathbb{N} .
$$


If we assume $\lambda_{1} \neq 1 \neq \lambda_{2}=\lambda_{3}$, then (83) and Lemma 2 imply

$$
\begin{aligned}
y_{n}= & \frac{\lambda_{1}^{2}\left(\lambda_{1}^{n}-1\right)}{\left(\lambda_{2}-\lambda_{1}\right)^{2}\left(\lambda_{1}-1\right)}+\frac{\left(\lambda_{2}-2 \lambda_{1}\right) \lambda_{2}\left(\lambda_{2}^{n}-1\right)}{\left(\lambda_{2}-\lambda_{1}\right)^{2}\left(\lambda_{2}-1\right)} \\
& +\frac{\lambda_{2}^{2}\left(1-n \lambda_{2}^{n-1}+(n-1) \lambda_{2}^{n}\right)}{\left(\lambda_{2}-\lambda_{1}\right)\left(\lambda_{2}-1\right)^{2}}, \quad n \in \mathbb{N} .
\end{aligned}
$$

Moreover, using (74) it is easily checked that (84) holds also for $n \geq-2$.

If we assume that $\lambda_{1} \neq 1$ and $\lambda_{2}=\lambda_{3}=1$, then from (83) it follows that

$$
y_{n}=\frac{\lambda_{1}^{2}\left(\lambda_{1}^{n}-1\right)}{\left(\lambda_{1}-1\right)^{3}}+\frac{\left(1-2 \lambda_{1}\right) n}{\left(\lambda_{1}-1\right)^{2}}+\frac{(n-1) n}{2\left(1-\lambda_{1}\right)} .
$$

Moreover, (85) holds also for $n \geq-2$. This case is obtained when $(a-1)(c-1)=b d$ and $2 a+c=3$, which implies

$$
P_{3}(\lambda)=\lambda^{3}-a \lambda^{2}+(2 a-3) \lambda+2-a=(\lambda-1)^{2}(\lambda+2-a)
$$

So that $\lambda_{1}=a-2$.

If we assume that $\lambda_{1}=1$ and $\lambda_{2}=\lambda_{3} \neq 1$, then from (83) it follows that

$$
y_{n}=\frac{n}{\left(\lambda_{2}-1\right)^{2}}+\frac{\left(\lambda_{2}-2\right) \lambda_{2}\left(\lambda_{2}^{n}-1\right)}{\left(\lambda_{2}-1\right)^{3}}+\frac{\lambda_{2}^{2}\left(1-n \lambda_{2}^{n-1}+(n-1) \lambda_{2}^{n}\right)}{\left(\lambda_{2}-1\right)^{3}} \text {. }
$$

Moreover, (86) holds also for $n \geq-2$.

If $\Delta=p=0$, then $\lambda_{1}=\lambda_{2}=\lambda_{3}$, so the general solution to (26) has the following form:

$$
a_{n}=\left(\hat{\beta}_{1}+\hat{\beta}_{2} n+\hat{\beta}_{3} n^{2}\right) \lambda_{1}^{n}, \quad n \in \mathbb{N}
$$

where $\hat{\beta}_{1}, \hat{\beta}_{2}$ and $\hat{\beta}_{3}$ are constants.

To find the solution to equation (26) satisfying (28) in this case, we can let $\lambda_{2} \rightarrow \lambda_{1}$ in (82) and obtain

$$
a_{n}=\frac{(n+1)(n+2)}{2} \lambda_{1}^{n}
$$

for $n \geq-2$, from which along with (30) we obtain

$$
y_{n}=\sum_{j=0}^{n-1} \frac{(j+1)(j+2)}{2} \lambda_{1}^{j}
$$

for every $n \in \mathbb{N}$. Moreover, (89) holds also for $n \geq-2$.

If we assume that $\lambda_{1}=\lambda_{2}=\lambda_{3} \neq 1$, then from (87) and Lemma 2 it follows that

$$
\begin{aligned}
y_{n} & =\frac{2 s_{n}^{(0)}+3 \lambda_{1} s_{n-1}^{(1)}+\lambda_{1} s_{n-1}^{(2)}}{2} \\
& =\frac{2-(n+1)(n+2) \lambda_{1}^{n}+2 n(n+2) \lambda_{1}^{n+1}-n(n+1) \lambda_{1}^{n+2}}{2\left(1-\lambda_{1}\right)^{3}}
\end{aligned}
$$

for every $n \in \mathbb{N}$. In fact, (90) holds also for $n \geq-2$. 
If we assume $\lambda_{1}=\lambda_{2}=\lambda_{3}=1$, then from (87) and some calculation, we obtain

$$
y_{n}=\sum_{j=0}^{n-1} \frac{(j+1)(j+2)}{2}=\frac{n(n+1)(n+2)}{6}
$$

for every $n \in \mathbb{N}$, which obviously holds for $j=-2,-1,0$ (for more details, see $[28,40]$ ).

Corollary 2 Assume that $a, b, c, d \in \mathbb{Z} \backslash\{0\}, \alpha, \beta, z_{-2}, z_{-1}, w_{-2}, w_{-1} \in \mathbb{C} \backslash\{0\}, a c \neq b d$ and $\Delta=0$. Then the following statements are true.

(a) If $(a-1)(c-1) \neq b d$ and $a^{2}+3 c \neq 0$, then the general solution to system (2) is given by (25) and (35), where $\left(a_{n}\right)_{n \geq-2}$ is given by (82), $\left(y_{n}\right)_{n \geq-2}$ is given by (84), while $\lambda_{j}$, $j=\overline{1,3}$ are given by (71).

(b) If $(a-1)(c-1)=b d, 2 a+c=3$ and $a \neq 3$, then the general solution to system (2) $i$ s given by (25) and (35), where $\left(a_{n}\right)_{n \geq-2}$ is given by (82) with $\lambda_{2}=1,\left(y_{n}\right)_{n \geq-2}$ is given by (85), and $\lambda_{1}=a-2$.

(c) If $(a-1)(c-1)=b d, 2 a+c \neq 3$, then the general solution to system (2) is given by (25) and (35), where $\left(a_{n}\right)_{n \geq-2}$ is given by (82) with $\lambda_{1}=1,\left(y_{n}\right)_{n \geq-2}$ is given by (86), $\lambda_{1}=1$, while $\lambda_{2,3}$ are given by (79).

(d) If $(a-1)(c-1) \neq b d$ and $a^{2}+3 c=0$, then the general solution to system (2) is given by (25) and (35), where $\left(a_{n}\right)_{n \geq-2}$ is given by (88), $\left(y_{n}\right)_{n \geq-2}$ is given by (90), while $\lambda_{j}=a / 3$, $j=\overline{1,3}$.

(e) If $(a-1)(c-1)=b d$ and $2 a+c=3$ and $a=3$, then the general solution to system (2) is given by (25) and (35), where $\left(a_{n}\right)_{n \geq-2}$ is given by (88) with $\lambda_{1}=1$, while $\left(y_{n}\right)_{n \geq-2}$ is given by (91).

Remark 3 Recall that equation (70) will have a zero equal to one if $(a-1)(c-1)=b d$. If $\lambda=m \in \mathbb{Z} \backslash\{0\}$ is a double zero of $P_{3}$, then it must be

$$
\begin{aligned}
& P_{3}(\lambda)=\lambda^{3}-a \lambda^{2}-c \lambda+c m+a m^{2}-m^{3} \\
& P_{3}^{\prime}(m)=3 m^{2}-2 a m-c=0 .
\end{aligned}
$$

From (93) we have that $c=3 m^{2}-2 a m$. Using this in (92), we get

$$
P_{3}(\lambda)=\lambda^{3}-a \lambda^{2}+\left(2 a m-3 m^{2}\right) \lambda+2 m^{3}-a m^{2}=(\lambda-m)^{2}(\lambda-a+2 m) .
$$

From this, and since $a c \neq b d$, it follows that $a \neq 2 m$. Since $P_{3}^{\prime \prime}(m) \neq 0$, we also have that it must be $a \neq 3 m$. Hence, for every $m \in \mathbb{Z} \backslash\{0\}$ and $a \in \mathbb{Z}$ such that $2 m \neq a \neq 3 m$, the polynomials in (94) are of type (70), and they have exactly two equal zeros $\lambda_{1}=\lambda_{2}=m \neq$ $\lambda_{3}=a-2 m$, and if additionally $m \neq 1$ and $a \neq 2 m+1$ hold, we see that the conditions of Corollary 2(a) are satisfied. For $m=1$ and $a \neq 3$, it is obtained that $P_{3}$ can have 1 as a double zero, which means that such polynomials satisfy the conditions of Corollary 2(b). For $a=2 m+1$, we get a polynomial which has a double zero different from 1 , which is its third zero. This means that such polynomials satisfy the conditions of Corollary 2(c).

The polynomial has three equal zeros only if $q=0$, which along with $\Delta=0$ implies $p=0$. Hence, we have $c=-a^{2} / 3$ and $8 a c=9 b d$, which implies that $c_{1}=a^{3} / 27$, so that

$$
P_{3}(\lambda)=\lambda^{3}-a \lambda^{2}+\frac{a^{2}}{3} \lambda-\frac{a^{3}}{27}=\left(\lambda-\frac{a}{3}\right)^{3} .
$$


Since $c$ must be an integer, it follows that $a=3 \hat{a}$ for some $\hat{a} \in \mathbb{Z}$, so that $c=-3 \hat{a}^{2}$ and $c_{1}=\hat{a}^{3}$. Hence, for each $\hat{a} \in \mathbb{Z} \backslash\{0\}$, these relations give polynomials of the form in (70) which have three equal zeros. This means that such polynomials satisfy the conditions of Corollary 2(d). When $a=3$, polynomial $P_{3}$ has 1 as a triple zero, which means that there is a polynomial satisfying the conditions of Corollary $2(\mathrm{e})$.

Before we formulate our next result, we want to say that the solution to the difference equation

$$
z_{n+2}+a z_{n+1}+b z_{n}=0, \quad n \in \mathbb{N}_{0},
$$

with initial values $z_{0}, z_{1} \in \mathbb{C}$, where $a \in \mathbb{C}$ and $b \in \mathbb{C} \backslash\{0\}$, is given by

$$
z_{n}=\frac{\lambda_{2} z_{0}-z_{1}}{\lambda_{2}-\lambda_{1}} \lambda_{1}^{n}+\frac{z_{1}-\lambda_{1} z_{0}}{\lambda_{2}-\lambda_{1}} \lambda_{2}^{n}
$$

if $\lambda_{1} \neq \lambda_{2}$, and

$$
z_{n}=\left(z_{1} n+\lambda_{1} z_{0}(1-n)\right) \lambda_{1}^{n-1}
$$

if $\lambda_{1}=\lambda_{2}$, where $\lambda_{1}$ and $\lambda_{2}$ are the zeros of the characteristic polynomial $P_{2}(\lambda)=\lambda^{2}+a \lambda+b$. This simple result is well known and can be essentially found in any book on difference equations and many papers $[6,8,25]$.

Theorem 5 Assume that $a, b, c, d \in \mathbb{Z}, a c=b d, c d \neq 0, \alpha, \beta \in \mathbb{C} \backslash\{0\}$ and $z_{-2}, z_{-1}, w_{-2}, w_{-1} \in$ $\mathbb{C} \backslash\{0\}$. Then the following statements hold.

(a) If $a^{2} \neq-4 c$ and $a+c \neq 1$, then the general solution to system (2) is given by the following formulas:

$$
\begin{aligned}
z_{n}= & \alpha^{\frac{1}{1-a-c}\left(a \frac{\left(\lambda_{2}-1\right) \lambda_{1}^{n+1}-\left(\lambda_{1}-1\right) \lambda_{2}^{n+1}}{\lambda_{1}-\lambda_{2}}+1-c\right)} \\
& \times \beta^{\frac{a c}{d(a+c-1)}\left(\frac{\left(\lambda_{2}-1\right) \lambda_{1}^{n+1}-\left(\lambda_{1}-1\right) \lambda_{2}^{n+1}}{\lambda_{2}-\lambda_{1}}-1\right)} z_{-2}^{a c} \frac{\lambda_{1}^{n}-\lambda_{2}^{n}}{\lambda_{1}-\lambda_{2}} \\
& \times z_{-1}^{a \frac{\lambda_{1}^{n+1}-\lambda_{2}^{n+1}}{\lambda_{1}-\lambda_{2}}} w_{-2}^{\frac{a c^{2}}{d}} \frac{\lambda_{1}^{n}-\lambda_{2}^{n}}{\lambda_{1}-\lambda_{2}} w_{-1}^{\frac{a c}{d}} \frac{\lambda_{1}^{n+1}-\lambda_{2}^{n+1}}{\lambda_{1}-\lambda_{2}}
\end{aligned}
$$

and

$$
\begin{aligned}
w_{n}= & \alpha^{\frac{d}{1-a-c}\left(\frac{\left(\lambda_{2}-1\right) \lambda_{1}^{n}-\left(\lambda_{1}-1\right) \lambda_{2}^{n}}{\lambda_{1}-\lambda_{2}}+1\right)} \beta^{\frac{1}{a+c-1}\left(c \frac{\left(\lambda_{2}-1\right) \lambda_{1}^{n}-\left(\lambda_{1}-1\right) \lambda_{2}^{n}}{\lambda_{2}-\lambda_{1}}+a-1\right)} \\
& \times z_{-2}^{c d \frac{\lambda_{1}^{n-1}-\lambda_{2}^{n-1}}{\lambda_{1}-\lambda_{2}}} z_{-1}^{d \frac{\lambda_{1}^{n}-\lambda_{2}^{n}}{\lambda_{1}-\lambda_{2}}} w_{-2}^{c^{2} \frac{\lambda_{1}^{n-1}-\lambda_{2}^{n-1}}{\lambda_{1}-\lambda_{2}}} w_{-1}^{c \frac{\lambda_{1}^{n}-\lambda_{2}^{n}}{\lambda_{1}-\lambda_{2}}}
\end{aligned}
$$

where

$$
\lambda_{1,2}=\frac{a+\sqrt{a^{2}+4 c}}{2} .
$$


(b) If $a^{2}=-4 c$ and $a+c \neq 1$, then the general solution to system (2) is given by the following formulas:

$$
\begin{aligned}
z_{n}= & \alpha^{\frac{1}{a+c-1}\left(\left(-(n+1) \lambda_{1}^{3}+(n+2) \lambda_{1}^{2}+c(n-1) \lambda_{1}-c n\right) \lambda_{1}^{n-1}+c-1\right)} \\
& \times \beta^{\frac{c}{d(a+c-1)}\left(\left(-(n+1) \lambda_{1}^{3}+(n+2) \lambda_{1}^{2}+c(n-1) \lambda_{1}-c n\right) \lambda_{1}^{n-1}-a\right)} z_{-2}^{\left(c(n-1)-(n+1) \lambda_{1}^{2}\right) \lambda_{1}^{n}} \\
& \times z_{-1}^{\left((n+2) \lambda_{1}^{2}-c n\right) \lambda_{1}^{n-1}} w_{-2}^{\frac{c}{d}\left(c(n-1)-(n+1) \lambda_{1}^{2}\right) \lambda_{1}^{n}} w_{-1}^{\frac{c}{d}\left((n+2) \lambda_{1}^{2}-c n\right) \lambda_{1}^{n-1}}
\end{aligned}
$$

and

$$
\begin{aligned}
w_{n}= & \alpha^{\frac{d}{a+c-1}\left(\left(\lambda_{1}+n\left(1-\lambda_{1}\right)\right) \lambda_{1}^{n-1}-1\right)} \beta^{\frac{1}{a+c-1}\left(c\left(\lambda_{1}+\left(1-\lambda_{1}\right) n\right) \lambda_{1}^{n-1}+a-1\right)} z_{-2}^{d(1-n) \lambda_{1}^{n}} \\
& \times z_{-1}^{d n \lambda_{1}^{n-1}} w_{-2}^{c(1-n) \lambda_{1}^{n}} w_{-1}^{c n \lambda_{1}^{n-1}}
\end{aligned}
$$

where

$$
\lambda_{1}=a / 2
$$

(c) If $a^{2} \neq-4 c$ and $a+c=1$, then the general solution to system (2) is given by the following formulas:

$$
\begin{aligned}
z_{n}= & \alpha^{\frac{a(a-1)^{n+1}-n(a-1)^{2}-3(a-1)+n+1}{(a-2)^{2}}} \\
& \times \beta^{\frac{-a(a-1)^{n+2}+n a(1-a)(2-a)+a(a-1)^{2}}{d(2-a)^{2}}} z_{-2}^{a(a-1) \frac{(a-1)^{n}-1}{2-a}} \\
& \times z_{-1}^{a \frac{(a-1)^{n+1}-1}{a-2}} w_{-2}^{\frac{a(a-1)^{2}\left((a-1)^{n}-1\right)}{d(a-2)}} w_{-1}^{\frac{a(a-1)\left((a-1)^{n+1}-1\right)}{d(2-a)}}
\end{aligned}
$$

and

$$
\begin{aligned}
w_{n}= & \alpha^{\frac{d\left((a-1)^{n}+n(1-a)+n-1\right)}{(a-2)^{2}}} \beta^{\frac{-(a-1)^{n+1}+(2-a)^{2}-1+a+n(1-a)(2-a)}{(2-a)^{2}}} z_{-2}^{d(a-1) \frac{(a-1)^{n-1}-1}{2-a}} \\
& \times z_{-1}^{d \frac{(a-1)^{n}-1}{a-2}} w_{-2}^{\frac{(a-1)^{2}\left((a-1)^{n-1}-1\right)}{a-2}} w_{-1}^{\frac{(a-1)\left((a-1)^{n}-1\right)}{2-a}} .
\end{aligned}
$$

(d) If $a^{2}=-4 c$ and $a+c=1$, then the general solution to system (2) is given by the following formulas:

$$
z_{n}=\alpha^{n^{2}+n+1} \beta^{\frac{n(n+1)}{2}} z_{-2}^{-2 n} z_{-1}^{2 n+2} w_{-2}^{-b n} w_{-1}^{b(n+1)}
$$

and

$$
w_{n}=\alpha^{\frac{d(n-1) n}{2}} \beta^{\frac{2+n-n^{2}}{2}} z_{-2}^{d(1-n)} z_{-1}^{d n} w_{-2}^{n-1} w_{-1}^{-n}
$$

Proof First note that in this case equalities (49)-(66) also hold. Since $a c=b d$ and $c \neq 0$, we have

$$
u_{n+2}=a u_{n+1}+c u_{n}+d
$$




$$
\begin{aligned}
& v_{n+2}=a v_{n+1}+c v_{n}+1-a, \\
& \alpha_{n+2}=a \alpha_{n+1}+c \alpha_{n}, \\
& \beta_{n+2}=a \beta_{n+1}+c \beta_{n}, \\
& \gamma_{n+2}=a \gamma_{n+1}+c \gamma_{n}, \\
& \delta_{n+2}=a \delta_{n+1}+c \delta_{n}
\end{aligned}
$$

for $n \in \mathbb{N}_{0}$, while operator $A$ defined in (67) becomes

$$
A\left(t_{n}\right)=t_{n+2}-a t_{n+1}-c t_{n} .
$$

Now, equation (68) is of the second order, so solvable in closed form. Consequently, (110)(113) are solvable, so that closed form formulas for $\alpha_{n}, \beta_{n}, \gamma_{n}, \delta_{n}$ can be found by employing the corresponding conditions in (39)-(44). Such obtained formulas along with (59)-(62) yield formulas for $a_{n}, b_{n}, c_{n}, d_{n}$. Further, (69) is also solvable, since a particular solution to the equation has the form

$$
t_{n}=\hat{d}_{0}+\hat{d}_{1} n+\hat{d}_{2} n^{2}
$$

for some constants $\hat{d}_{j}, j=\overline{0,2}$. Specially, it is solved for $f=d$ and $f=1-a$, which gives formulas for the sequences $u_{n}$ and $v_{n}$, and consequently closed form formulas for $x_{n}$ and $y_{n}$. Using such obtained formulas in (47) and (48), we get formulas for solutions to system (2) in this case.

(a) Case $a^{2}+4 c \neq 0, a+c \neq 1$. In this case, a particular solution to equation (108) is

$$
u_{n}^{p}=\frac{d}{1-a-c}
$$

so that

$$
u_{n}=c_{1} \lambda_{1}^{n}+c_{2} \lambda_{2}^{n}+\frac{d}{1-a-c}, \quad n \in \mathbb{N}_{0}
$$

where

$$
\lambda_{1,2}=\frac{a \pm \sqrt{a^{2}+4 c}}{2},
$$

from which along with $u_{0}=u_{1}=0$ it follows that

$$
c_{1}=\frac{d\left(\lambda_{2}-1\right)}{(a+c-1)\left(\lambda_{2}-\lambda_{1}\right)} \quad \text { and } \quad c_{2}=\frac{d\left(1-\lambda_{1}\right)}{(a+c-1)\left(\lambda_{2}-\lambda_{1}\right)} \text {, }
$$

and consequently

$$
u_{n}=\frac{d}{1-a-c}\left(\frac{\left(\lambda_{2}-1\right) \lambda_{1}^{n}-\left(\lambda_{1}-1\right) \lambda_{2}^{n}}{\lambda_{1}-\lambda_{2}}+1\right) .
$$


Using (116) in (55), we get

$$
x_{n}=\frac{1}{1-a-c}\left(\frac{\left(\lambda_{2}-1\right)\left(\lambda_{1}^{2}-c\right) \lambda_{1}^{n}-\left(\lambda_{1}-1\right)\left(\lambda_{2}^{2}-c\right) \lambda_{2}^{n}}{\lambda_{1}-\lambda_{2}}+1-c\right) .
$$

Particular solution to equation (109) in this case is

$$
v_{n}^{p}=\frac{1-a}{1-a-c},
$$

so that

$$
v_{n}=d_{1} \lambda_{1}^{n}+d_{2} \lambda_{2}^{n}+\frac{1-a}{1-a-c}, \quad n \in \mathbb{N}_{0},
$$

where $\lambda_{1,2}$ are given by (115), from which along with $v_{0}=v_{1}=1$ it follows that

$$
d_{1}=\frac{c\left(\lambda_{2}-1\right)}{(a+c-1)\left(\lambda_{2}-\lambda_{1}\right)} \quad \text { and } \quad d_{2}=\frac{c\left(1-\lambda_{1}\right)}{(a+c-1)\left(\lambda_{2}-\lambda_{1}\right)}
$$

and consequently

$$
v_{n}=\frac{1}{a+c-1}\left(c \frac{\left(\lambda_{2}-1\right) \lambda_{1}^{n}-\left(\lambda_{1}-1\right) \lambda_{2}^{n}}{\lambda_{2}-\lambda_{1}}+a-1\right) .
$$

Using (119) in (57), we get

$$
y_{n}=\frac{c}{d(a+c-1)}\left(\frac{\left(\lambda_{2}-1\right)\left(\lambda_{1}^{2}-c\right) \lambda_{1}^{n}-\left(\lambda_{1}-1\right)\left(\lambda_{2}^{2}-c\right) \lambda_{2}^{n}}{\lambda_{2}-\lambda_{1}}-a\right) .
$$

By using the corresponding conditions in (43)-(46) in formula (96), we get

$$
\begin{aligned}
& \alpha_{n}=d \lambda_{1} \lambda_{2} \frac{\lambda_{1}^{n-1}-\lambda_{2}^{n-1}}{\lambda_{2}-\lambda_{1}}, \\
& \beta_{n}=d \frac{\lambda_{1}^{n}-\lambda_{2}^{n}}{\lambda_{1}-\lambda_{2}} \\
& \gamma_{n}=c \lambda_{1} \lambda_{2} \frac{\lambda_{1}^{n-1}-\lambda_{2}^{n-1}}{\lambda_{2}-\lambda_{1}}, \\
& \delta_{n}=c \frac{\lambda_{1}^{n}-\lambda_{2}^{n}}{\lambda_{1}-\lambda_{2}}
\end{aligned}
$$

By using (121)-(124) in (59)-(62), we get

$$
\begin{aligned}
& a_{n}=\lambda_{1} \lambda_{2} \frac{\left(\lambda_{1}^{2}-c\right) \lambda_{1}^{n-1}-\left(\lambda_{2}^{2}-c\right) \lambda_{2}^{n-1}}{\lambda_{2}-\lambda_{1}}, \\
& b_{n}=\frac{\left(\lambda_{1}^{2}-c\right) \lambda_{1}^{n}-\left(\lambda_{2}^{2}-c\right) \lambda_{2}^{n}}{\lambda_{1}-\lambda_{2}}, \\
& c_{n}=\frac{c \lambda_{1} \lambda_{2}}{d} \frac{\left(\lambda_{1}^{2}-c\right) \lambda_{1}^{n-1}-\left(\lambda_{2}^{2}-c\right) \lambda_{2}^{n-1}}{\lambda_{2}-\lambda_{1}}, \\
& d_{n}=\frac{c}{d} \frac{\left(\lambda_{1}^{2}-c\right) \lambda_{1}^{n}-\left(\lambda_{2}^{2}-c\right) \lambda_{2}^{n}}{\lambda_{1}-\lambda_{2}} .
\end{aligned}
$$


Using (116), (117), (119)-(128) in (47) and (48), as well as the fact that $\lambda_{1,2}$ are roots of the polynomial $\lambda^{2}-a \lambda-c$, and after some standard calculations, formulas (98) and (99) are obtained.

(b) Case $a^{2}+4 c=0, a+c \neq 1$. In this case, we have $\lambda_{1}=\lambda_{2} \neq 1$ and a particular solution to equation (108) is given by (114), so that

$$
u_{n}=\left(c_{1}+c_{2} n\right) \lambda_{1}^{n}+\frac{d}{1-a-c}, \quad n \in \mathbb{N}_{0}
$$

where $\lambda_{1}$ is given by (103), from which along with $u_{0}=u_{1}=0$ it follows that

$$
c_{1}=\frac{d}{a+c-1} \quad \text { and } \quad c_{2}=\frac{d\left(1-\lambda_{1}\right)}{(a+c-1) \lambda_{1}}
$$

and consequently

$$
u_{n}=\frac{d}{a+c-1}\left(\left(\lambda_{1}+n\left(1-\lambda_{1}\right)\right) \lambda_{1}^{n-1}-1\right) .
$$

Using (129) in (55), we get

$$
x_{n}=\frac{1}{a+c-1}\left(\left(-(n+1) \lambda_{1}^{3}+(n+2) \lambda_{1}^{2}+c(n-1) \lambda_{1}-c n\right) \lambda_{1}^{n-1}+c-1\right)
$$

Particular solution to equation (109) in this case is given by (118), so that

$$
v_{n}=\left(d_{1}+d_{2} n\right) \lambda_{1}^{n}+\frac{1-a}{1-a-c}, \quad n \in \mathbb{N}_{0},
$$

where $\lambda_{1}$ is given by (103), from which along with $v_{0}=v_{1}=1$ it follows that

$$
d_{1}=\frac{c}{a+c-1} \quad \text { and } \quad d_{2}=\frac{c\left(1-\lambda_{1}\right)}{(a+c-1) \lambda_{1}}
$$

and consequently

$$
v_{n}=\frac{1}{a+c-1}\left(c\left(\lambda_{1}+\left(1-\lambda_{1}\right) n\right) \lambda_{1}^{n-1}+a-1\right) .
$$

Using (131) in (57), we get

$$
y_{n}=\frac{c}{d(a+c-1)}\left(\left(-(n+1) \lambda_{1}^{3}+(n+2) \lambda_{1}^{2}+c(n-1) \lambda_{1}-c n\right) \lambda_{1}^{n-1}-a\right) .
$$

By using the corresponding conditions in (43)-(46) in formula (97), we get

$$
\begin{aligned}
& \alpha_{n}=d(1-n) \lambda_{1}^{n}, \\
& \beta_{n}=d n \lambda_{1}^{n-1}, \\
& \gamma_{n}=c(1-n) \lambda_{1}^{n}, \\
& \delta_{n}=c n \lambda_{1}^{n-1} .
\end{aligned}
$$


By using (133)-(136) in (59)-(62), we get

$$
\begin{aligned}
& a_{n}=\left(c(n-1)-(n+1) \lambda_{1}^{2}\right) \lambda_{1}^{n}, \\
& b_{n}=\left((n+2) \lambda_{1}^{2}-c n\right) \lambda_{1}^{n-1}, \\
& c_{n}=\frac{c}{d}\left(c(n-1)-(n+1) \lambda_{1}^{2}\right) \lambda_{1}^{n}, \\
& d_{n}=\frac{c}{d}\left((n+2) \lambda_{1}^{2}-c n\right) \lambda_{1}^{n-1} .
\end{aligned}
$$

Using the equalities in (129)-(140), in (47) and (48), formulas (101) and (102) are obtained.

(c) Case $a^{2}+4 c \neq 0, a+c=1$. In this case, we have that $\lambda_{1} \neq 1=\lambda_{2}$ and that a particular solution to equation (108) is given by

$$
u_{n}^{p}=\frac{d n}{2-a}
$$

so that

$$
u_{n}=c_{1} \lambda_{1}^{n}+c_{2}+\frac{d n}{2-a}, \quad n \in \mathbb{N}_{0},
$$

where $\lambda_{1}=-c=a-1$, from which along with $u_{0}=u_{1}=0$ it follows that

$$
c_{1}=-c_{2}=\frac{d}{(a-2)\left(\lambda_{1}-1\right)}=\frac{d}{(a-2)^{2}},
$$

and consequently

$$
\begin{aligned}
u_{n} & =\frac{d}{a-2}\left(\frac{\lambda_{1}^{n}-1}{\lambda_{1}-1}-n\right)=\frac{d\left(\lambda_{1}^{n}-n \lambda_{1}+n-1\right)}{(a-2)^{2}} \\
& =\frac{d\left((a-1)^{n}+n(1-a)+n-1\right)}{(a-2)^{2}} .
\end{aligned}
$$

Using (142) in (55), we get

$$
x_{n}=\frac{a(a-1)^{n+1}-n(a-1)^{2}-3(a-1)+n+1}{(a-2)^{2}} .
$$

Particular solution to equation (109) in this case is given by

$$
v_{n}^{p}=\frac{n(1-a)}{2-a},
$$

so that

$$
v_{n}=d_{1} \lambda_{1}^{n}+d_{2}+\frac{n(1-a)}{2-a}, \quad n \in \mathbb{N}_{0},
$$

from which along with $v_{0}=v_{1}=1$ it follows that

$$
d_{1}=\frac{1-a}{(2-a)^{2}} \quad \text { and } \quad d_{2}=1-\frac{1-a}{(2-a)^{2}}
$$


and consequently

$$
v_{n}=\frac{-(a-1)^{n+1}+(2-a)^{2}-1+a+n(1-a)(2-a)}{(2-a)^{2}} .
$$

Using (145) in (57), we get

$$
y_{n}=\frac{-a(a-1)^{n+2}+n a(1-a)(2-a)+a(a-1)^{2}}{d(2-a)^{2}} .
$$

By using the corresponding conditions in (43)-(46) in formula (96), we get

$$
\begin{aligned}
& \alpha_{n}=d \lambda_{1} \frac{\lambda_{1}^{n-1}-1}{1-\lambda_{1}}=d(a-1) \frac{(a-1)^{n-1}-1}{2-a}, \\
& \beta_{n}=d \frac{\lambda_{1}^{n}-1}{\lambda_{1}-1}=d \frac{(a-1)^{n}-1}{a-2}, \\
& \gamma_{n}=c \lambda_{1} \frac{\lambda_{1}^{n-1}-1}{1-\lambda_{1}}=(a-1)^{2} \frac{(a-1)^{n-1}-1}{a-2}, \\
& \delta_{n}=c \frac{\lambda_{1}^{n}-1}{\lambda_{1}-1}=(a-1) \frac{(a-1)^{n}-1}{2-a} .
\end{aligned}
$$

By using (147)-(150) in (59)-(62), we get

$$
\begin{aligned}
& a_{n}=a(a-1) \frac{(a-1)^{n}-1}{2-a}, \\
& b_{n}=a \frac{(a-1)^{n+1}-1}{a-2}, \\
& c_{n}=\frac{a(a-1)^{2}\left((a-1)^{n}-1\right)}{d(a-2)}, \\
& d_{n}=\frac{a(a-1)\left((a-1)^{n+1}-1\right)}{d(2-a)} .
\end{aligned}
$$

Using (142), (143), (145)-(154) in (47) and (48), formulas (104) and (105) are obtained.

(d) Case $a^{2}+4 c=0, a+c=1$. In this case, we have that $a=2$ and $c=-1$. We also have that $\lambda_{1}=\lambda_{2}=1$, and that a particular solution to equation (108) is given by

$$
u_{n}^{p}=\frac{d n^{2}}{2}
$$

so that

$$
u_{n}=c_{1}+c_{2} n+\frac{d n^{2}}{2}, \quad n \in \mathbb{N}_{0}
$$

from which along with $u_{0}=u_{1}=0$ it follows that $c_{1}=0$ and $c_{2}=-\frac{d}{2}$, and consequently

$$
u_{n}=\frac{d(n-1) n}{2}
$$


Using (156) in (55), we get

$$
x_{n}=n^{2}+n+1
$$

Particular solution to equation (109) in this case is given by

$$
v_{n}^{p}=-\frac{n^{2}}{2}
$$

so that

$$
v_{n}=d_{1}+d_{2} n-\frac{n^{2}}{2}, \quad n \in \mathbb{N}_{0}
$$

from which along with $v_{0}=v_{1}=1$ it follows that $d_{1}=1$ and $d_{2}=1 / 2$, and consequently

$$
v_{n}=\frac{2+n-n^{2}}{2} .
$$

Using (159) in (57), we get

$$
y_{n}=-\frac{n(n+1)}{d}=\frac{b n(n+1)}{2} .
$$

By using the corresponding conditions in (43)-(46) in formula (97), we get

$$
\begin{aligned}
& \alpha_{n}=d(1-n), \\
& \beta_{n}=d n, \\
& \gamma_{n}=n-1, \\
& \delta_{n}=-n .
\end{aligned}
$$

By using (161)-(164) in (59)-(62), we get

$$
\begin{aligned}
& a_{n}=-2 n, \\
& b_{n}=2 n+2, \\
& c_{n}=\frac{2 n}{d}=-b n, \\
& d_{n}=-\frac{2(n+1)}{d}=b(n+1) .
\end{aligned}
$$

Using (156), (157), (159)-(168) in (47) and (48), formulas (106) and (107) are obtained. By some calculation it can be proved that formulas (99)-(107) really satisfy system (2), which was checked by the author.

The author declares that he has no competing interests. 


\section{Publisher's Note}

Springer Nature remains neutral with regard to jurisdictional claims in published maps and institutional affiliations.

Received: 25 April 2017 Accepted: 11 May 2017 Published online: 26 May 2017

\section{References}

1. Agarwal, RP: Difference Equations and Inequalities: Theory, Methods, and Applications, 2nd edn. Dekker, New York (2000)

2. Andruch-Sobilo, A, Migda, M: Further properties of the rational recursive sequence $x_{n+1}=a x_{n-1} /\left(b+c x_{n} x_{n-1}\right)$. Opusc Math. 26(3), 387-394 (2006)

3. Andruch-Sobilo, A, Migda, M: On the rational recursive sequence $x_{n+1}=a x_{n-1} /\left(b+c x_{n} x_{n-1}\right)$. Tatra Mt. Math. Publ. 43, 1-9 (2009)

4. Berg, L, Stević, S: On some systems of difference equations. Appl. Math. Comput. 218, 1713-1718 (2011)

5. Jordan, C: Calculus of Finite Differences. Chelsea, New York (1956)

6. Krechmar, VA: A Problem Book in Algebra. Mir, Moscow (1974)

7. Levy, H, Lessman, F: Finite Difference Equations. Dover, New York (1992)

8. Mitrinović, DS, Kečkić, JD: Methods for Calculating Finite Sums. Naučna Knjiga, Beograd (1984) (in Serbian)

9. Papaschinopoulos, G, Fotiades, N, Schinas, CJ: On a system of difference equations including negative exponential terms. J. Differ. Equ. Appl. 20(5-6), 717-732 (2014)

10. Papaschinopoulos, G, Psarros, N, Papadopoulos, KB: On a system of $m$ difference equations having exponential terms. Electron. J. Qual. Theory Differ. Equ. 2015, Article ID 5 (2015)

11. Papaschinopoulos, $G$, Radin, $M$, Schinas, CJ: On the system of two difference equations of exponential form $x_{n+1}=a+b x_{n-1} e^{-y_{n}}, y_{n+1}=c+d y_{n-1} e^{-x_{n}}$. Math. Comput. Model. 54(11-12), 2969-2977 (2011)

12. Papaschinopoulos, G, Schinas, CJ: On a system of two nonlinear difference equations. J. Math. Anal. Appl. 219(2), 415-426 (1998)

13. Papaschinopoulos, G, Schinas, CJ: On the behavior of the solutions of a system of two nonlinear difference equations. Commun. Appl. Nonlinear Anal. 5(2), 47-59 (1998)

14. Papaschinopoulos, G, Schinas, CJ: Invariants for systems of two nonlinear difference equations. Differ. Equ. Dyn. Syst. 7(2), 181-196 (1999)

15. Papaschinopoulos, G, Schinas, CJ: Invariants and oscillation for systems of two nonlinear difference equations. Nonlinear Anal. TMA 46(7), 967-978 (2001)

16. Papaschinopoulos, G, Schinas, CJ: On the dynamics of two exponential type systems of difference equations. Comput. Math. Appl. 64(7), 2326-2334 (2012)

17. Papaschinopoulos, G, Stefanidou, G: Asymptotic behavior of the solutions of a class of rational difference equations Int. J. Difference Equ. 5(2), 233-249 (2010)

18. Stefanidou, G, Papaschinopoulos, G, Schinas, C: On a system of max difference equations. Dyn. Contin. Discrete Impuls. Syst. Ser. A 14(6), 885-903 (2007)

19. Stefanidou, G, Papaschinopoulos, G, Schinas, CJ: On a system of two exponential type difference equations. Commun. Appl. Nonlinear Anal. 17(2), 1-13 (2010)

20. Stević, S: On a generalized max-type difference equation from automatic control theory. Nonlinear Anal. TMA 72, 1841-1849 (2010)

21. Stević, S: On the difference equation $x_{n}=x_{n-k} /\left(b+c x_{n-1} \cdots x_{n-k}\right)$. Appl. Math. Comput. 218, 6291-6296 (2012)

22. Stević, S: Solutions of a max-type system of difference equations. Appl. Math. Comput. 218, 9825-9830 (2012)

23. Stević, S: Domains of undefinable solutions of some equations and systems of difference equations. Appl. Math. Comput. 219, 11206-11213 (2013)

24. Stević, S: First-order product-type systems of difference equations solvable in closed form. Electron. J. Differ. Equ. 2015, Article ID 308 (2015)

25. Stević, S: Representation of solutions of bilinear difference equations in terms of generalized Fibonacci sequences. Electron. J. Qual. Theory Differ. Equ. 2014, Article ID 67 (2014)

26. Stević, S: Product-type system of difference equations of second-order solvable in closed form. Electron. J. Qual. Theory Differ. Equ. 2015, Article ID 56 (2015)

27. Stević, S: New solvable class of product-type systems of difference equations on the complex domain and a new method for proving the solvability. Electron. J. Qual. Theory Differ. Equ. 2016, Article ID 120 (2016)

28. Stević, S: Solvability of a product-type system of difference equations with six parameters. Adv. Nonlinear Anal. (in press). doi:10.1515/anona-2016-0145

29. Stević, S: Solvable subclasses of a class of nonlinear second-order difference equations. Adv. Nonlinear Anal. 5(2), 147-165 (2016)

30. Stević, S: Solvable product-type system of difference equations whose associated polynomial is of the fourth order Electron. J. Qual. Theory Differ. Equ. 2017, Article ID 13 (2017)

31. Stević, S, Alghamdi, MA, Alotaibi, A, Elsayed, EM: Solvable product-type system of difference equations of second order. Electron. J. Differ. Equ. 2015, Article ID 169 (2015)

32. Stević, S, Alghamdi, MA, Alotaibi, A, Shahzad, N: Boundedness character of a max-type system of difference equations of second order. Electron. J. Qual. Theory Differ. Equ. 2014, Article ID 45 (2014)

33. Stević, S, Diblik, J, Iričanin, B, Šmarda, Z: On a third-order system of difference equations with variable coefficients. Abstr. Appl. Anal. 2012, Article ID 508523 (2012)

34. Stević, S, Diblik, J, Iričanin, B, Šmarda, Z: On some solvable difference equations and systems of difference equations. Abstr. Appl. Anal. 2012, Article ID 541761 (2012)

35. Stević, S, Diblik, J, Iričanin, B, Šmarda, Z: On a solvable system of rational difference equations. J. Differ. Equ. Appl. 20(5-6), 811-825 (2014)

36. Stević, S, Diblik, J, Iričanin, B, Šmarda, Z: Solvability of nonlinear difference equations of fourth order. Electron. J. Differ Equ. 2014, Article ID 264 (2014)

37. Stević, S, Iričanin, B, Šmarda, Z: On a product-type system of difference equations of second order solvable in closed form. J. Inequal. Appl. 2015, Article ID 327 (2015) 
38. Stević, S, Iričanin, B, Šmarda, Z: Solvability of a close to symmetric system of difference equations. Electron. J. Differ. Equ. 2016, Article ID 159 (2016)

39. Stević, S, Iričanin, B, Šmarda, Z: Two-dimensional product-type system of difference equations solvable in closed form. Adv. Differ. Equ. 2016, Article ID 253 (2016)

40. Stević, S, Ranković, D: On a practically solvable product-type system of difference equations of second order. Electron. J. Qual. Theory Differ. Equ. 2016, Article ID 56 (2016)

41. Faddeyev, DK: Lectures on Algebra. Nauka, Moscow (1984) (in Russian)

Submit your manuscript to a SpringerOpen ${ }^{\circ}$ journal and benefit from:

- Convenient online submission

- Rigorous peer review

- Immediate publication on acceptance

- Open access: articles freely available online

- High visibility within the field

- Retaining the copyright to your article 